\title{
A Case of Steven-Johnson Syndrome: Favipiravir induced or Covid-19 disease related?
}

\author{
Icim Komurcugil $^{1}$, Nermin Karaosmanoglu ${ }^{1}$, Burak Engin ${ }^{1}$, and Ozlem Tanas Isikci ${ }^{1}$ \\ ${ }^{1}$ Ankara Eğitim Ve Araştırma Hastanesi
}

October 20, 2021

\begin{abstract}
Coronavirus disease 2019 (COVID-19) is caused by Severe Acute Respiratory Syndrome- Coronavirus-2 (SARS-CoV-2) and has affected millions of individuals. Here, we report the case of a 57-year-old man, who had COVID-19 infection and was treated with favipravir. He came to our clinic with erosions in oral and anogenital region.
\end{abstract}

\section{Introduction}

The international outbreak of respiratory illness termed coronavirus disease 2019 (COVID-19) began in December 2019 and has affected millions of individuals worlwide. There are various therapeutic agents approved by FDA for COVID-19. For instance, favipiravir is reported as one of the possible medications for shortening the duration of COVID-19 symptoms, by reducing inflammation. Here, we report the case of a 57-year-old man presented with fever and dry cough, who had COVID-19 infection and later presented with erosions in oral, anogenital regions and dorsum of his hands.

Case

A 57 - year- old male patient who had been diagnosed with Covid-19 on 21 st of December 2020,was consulted from Emergency Department to Dermatology department. He presented with painful erosions in the oral mucosa and anogenital area. [Figure 1] He was asymptomatic for Covid- 19 and was under treatment favipiravir.

We got a 4mm punch biopsy with 'Steven Johnson Syndrome (SJS)' and generalized bullous drug eruption as the initial diagnosis. In the biopsy specimen; subepidermal blistering due to marked epidermal necrosis was reported. Dermis and epidermis were distincly dissociated. There were fibrin and mixed inflammatory cells located in the subepidermal cleft. [Figure 2] The dermis was infiltrated with moderate cell infiltration, mostly with eosinophils and vascular proliferation.

The biopsy was consistent with SJS. Together with the clinical and histopathological findings, the patients was diagnosed as SJS. The patient was treated with $60 \mathrm{mg}$ intramuscular methylprednisolone for 3 days and topical silver sulfadiazin twice a day. In addition, the patient applied wet dressing 5 times a day with $0.1 \%$ Rivanol solution. After 1 -week-treatment period, the patient almost completely got healed. [Figure 3]

\section{Discussion}

SJS is a rare adverse drug reaction with a high mortality rate and multi- organ failure. This syndrome is characterized by dusty patches, atypical targetoid lesions, bullae and erosions and epidermal detachment less than $10 \%$. It occurs more often in women unlike our case.

There are various mechanisms by which this syndrome occurs. First of all, after the responsible drug is taken, the drug itself binds to T-cell receptors or MHC class 1 receptors in antigen-presenting-cells (APC). 
The result is clonal expansion of CD8+ cytotoxic T cells and Natural Killer Cells (NK Cells); leading to epidermal necrosis. Fas-FasL, expressed on the activated cytotoxic T cells, can also destroy keratinocytes via the production of intracellular caspases. In addition, it is thought that nitrous oxide (NO) induced by tumor necrosis factor- $\alpha$ (TNF- $\alpha$ ) and interferon (IFN)-alpha may also stimulate caspases.[1] Moreover, recent studies have shown high levels of cytotoxic molecules within lesions of SJS/TEN, such as perforin, granzyme B, and granulysin. [2]

SJS may be caused by either drugs or infections. The most frequently associated drugs are reported as allopurinol, aminopenicillins, cephalosporins, anticonvulsants and NSAIDs. [3] To our knowledge, there is no reported SJS case caused by favipiravir, in the literature. In our case; it is not clear whether the lesions of the patient are caused by Covid-19 infection or by the use of favipiravir.

There are various skin manifestations of COVID-19 reported in the recent literature. Those findings include urticaria, pityriasis rosea-like eruption, chickenpox-like vesicles and chillblain-like lesions.[4] Moreover, SJSlike lesions may be a manifestation of COVID-19 infection, as well. Up to now, there are a few case reports in the literature indicating that Covid-19 disease may be related with SJS. Parlakay et al. reported a case of a pediatric patient, with COVID-19 presenting like SJS. In their case, either amoxicillin-clavulonate treatment given for tonsillitis or COVID-19 infection could be the cause of SJS, as in our case. [5] In addition, it is discussed in a case-report that viral infections such as COVID-19 might induce SJS and susceptibility to drugs may be increased in patients with COVID-19 infection. [6] Despite the fact that, SJS has been linked to drug exposure, infectious agents such as HIV , Mycoplasma pneumonia, Herpes Simplex virus may have an effect on the incidence of SJS. [2] In fact, there is still no evidence that Covid-19 infection is a cause of SJS. We wanted to indicate that, SJS - a very serious adverse drug reaction -may be triggered by either Covid-19 - the very common disease all over the world - or by the very commonly used drug, favipiravir.

\section{References}

1) Oakley AM, Krishnamurthy K. Stevens Johnson Syndrome. In: StatPearls . Treasure Island (FL): StatPearls Publishing; April 19, 2021.

2) Su SC, Chung WH. Update on pathobiology in Stevens-Johnson syndrome and toxic epidermal necrolysis. Dermatologica Sinica. 2013;31(4), 175-180. doi:10.1016/j.dsi.2013.09.002.

3) Harr T, French LE. Toxic epidermal necrolysis and Stevens-Johnson syndrome. Orphanet J Rare Dis . 2010;5:39. Published 2010 Dec 16. doi:10.1186/1750-1172-5-39.

4) Singh H, Kaur H, Singh K, Sen CK. Cutaneous Manifestations of COVID-19: A Systematic Review. Adv Wound Care (New Rochelle) . 2021;10(2):51-80. doi:10.1089/wound.2020.1309

5) Parlakay A, Ozcan S, Karaatmaca B, Tiftik M. COVID-19 presenting like Steven Johnson Syndrome in a pediatric patient. Authorea . 2020 doi: 10.22541/au.159286119.92728253.

6) Bulat V, Likic R, Pondeljak N, Azdajic MD. Pholcodine-induced Stevens-Johnson syndrome in a patient with COVID-19 [published online ahead of print, 2021 Apr 20]. Postgrad Med J . 2021;postgradmedj-2021139892. doi:10.1136/postgradmedj-2021-139892

\section{Figure Legends}

Figure 1. erosions in the oral mucosa and genital area

Figure 2. fibrin and mixed inflammatory cells located in the subepidermal cleft

Figure 3. healed oral mucosa after treatment 

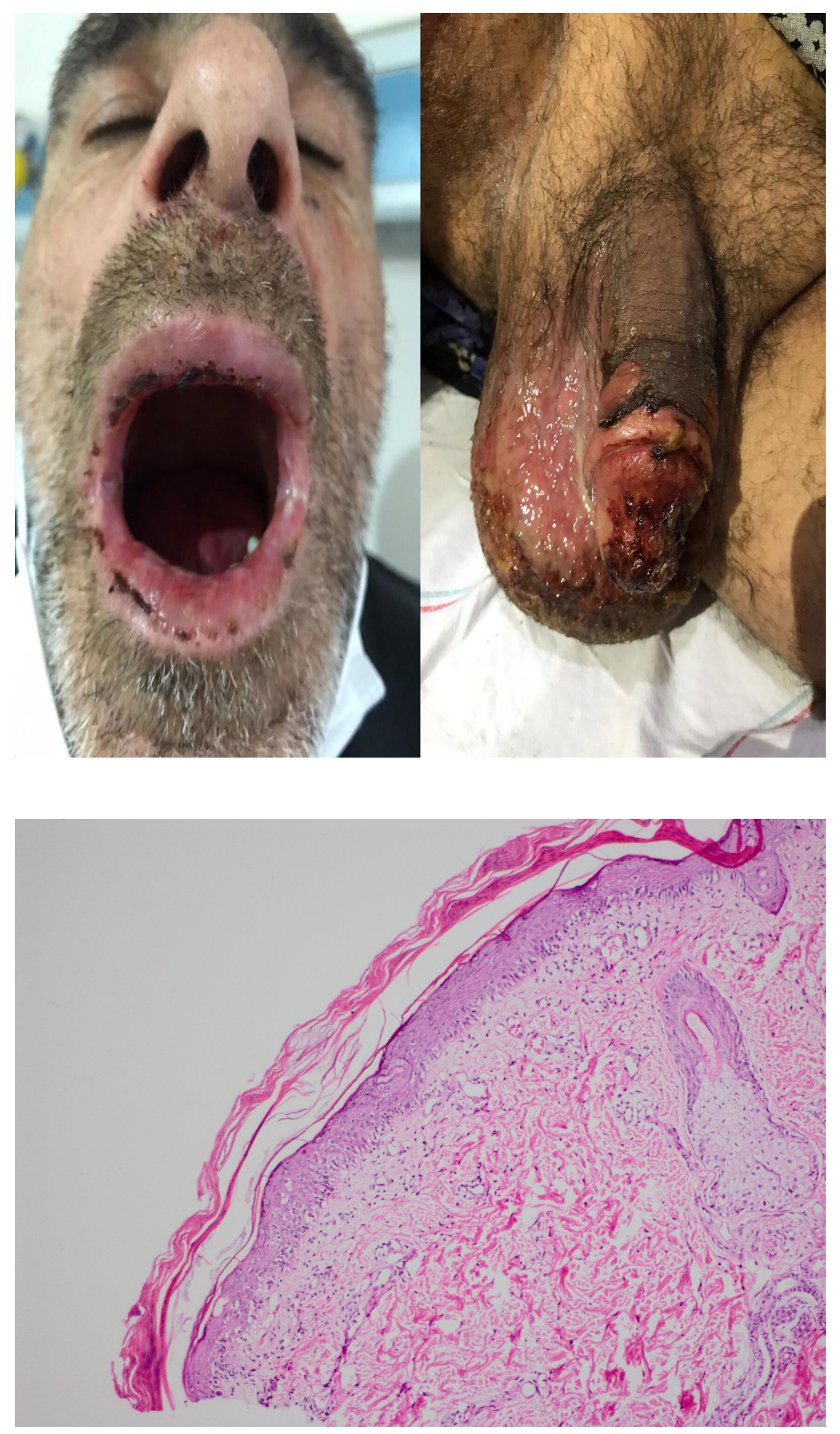


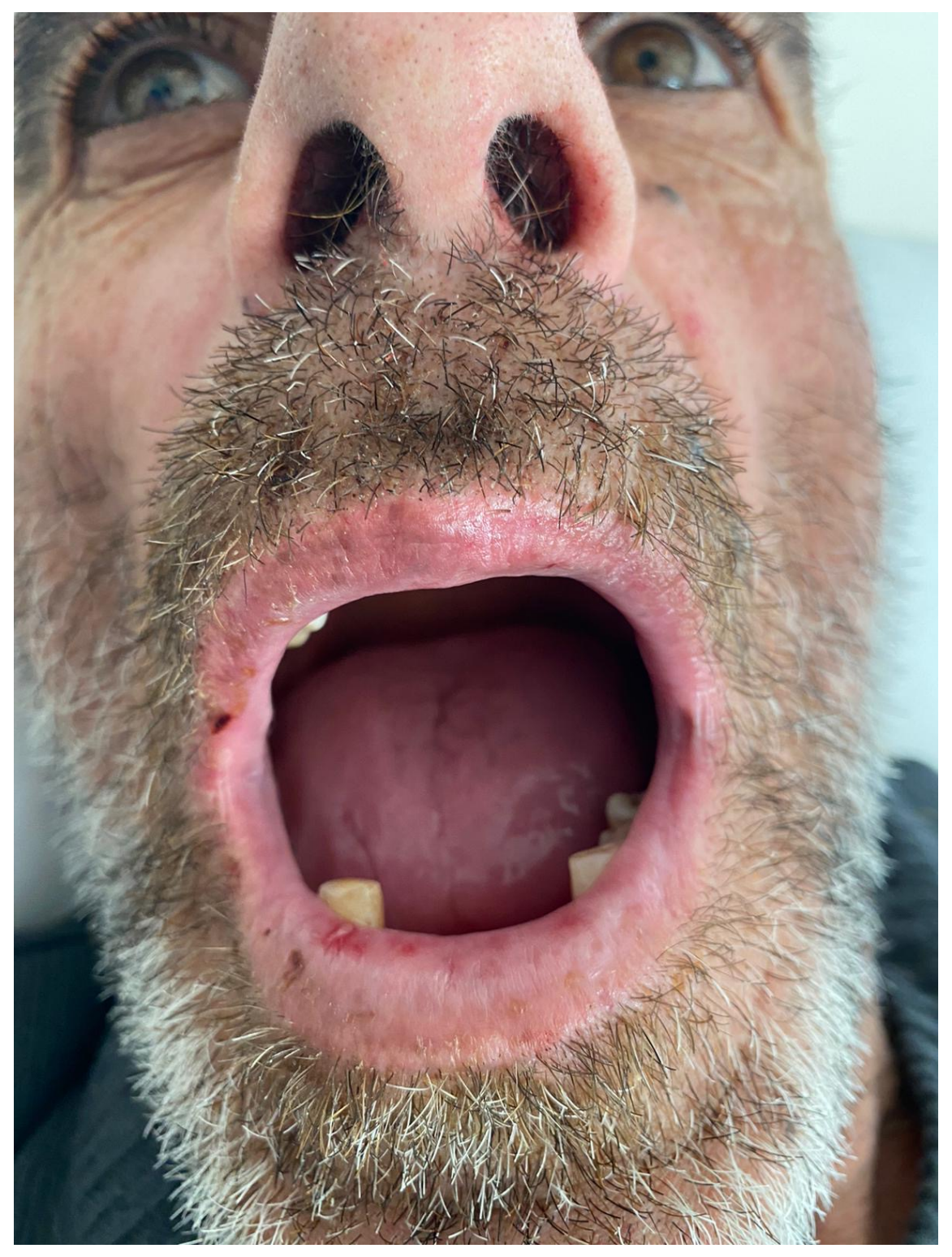

